\title{
THE BESSY SOFT X-RAY FEL USER FACILITY*
}

\section{Krämer ${ }^{\#}$ on behalf of the BESSY-FEL Design Group, BESSY, Berlin, Germany.}

\section{Abstract}

Free Electron Lasers will be the future light sources to generate intense ultra-short photon pulses. The user requests for an optimized $2^{\text {nd }}$ generation FEL facility in the VUV to soft X-ray range demands for ultra short photon pulses $(\Delta \mathrm{t} \cdot 20 \mathrm{fs})$ at a peak power of several $\mathrm{GW}$. Achieving a high shot to shot reproducibility of the pulse shape and pulse power will be mandatory and is feasible in a seeded High Gain Harmonic Generation (HGHG) approach.

Freely selectable photon polarization and wavelength tuning is essential for the new light sources as the proposed BESSY-Soft X-ray FEL user facility. Variable pulse repetition rates and alterable pulse patterns, including fast switching of the full bunch train or single bunches to different parallel operating FEL-Lines thus are foreseen for the facility.

BESSY's recently published Technical Design Report for a multi-user facility[1] is based on a cascaded HGHGscheme[2]. The combination of a superconducting $\mathrm{CW}$ linac, based on the TESLA-development, and the stability and reproducibility of the HGHG-scheme together with frequency and polarization tuning using gap-variable APPLE III-type undulators are the key elements for the proposal presently under evaluation by the funding agency. The status of the BESSY HGHG-FEL project is reviewed in the paper.

\section{INTRODUCTION}

Since 1999 BESSY is operating a $3^{\text {rd }}$ generation light source, BESSY II, serving for experiments in the VUV to soft X-ray range; for metrology application a new source, optimized for the EUV spectral regime, the Metrology Light source (MLS) is under construction[3]. As femtosecond time-resolved experiments are of increasing interest[4], a femto-slicing X-ray source was commissioned recently at the BESSY storage ring[5]. Though the sub-100 fs pulses are more than 2 orders in magnitude shorter than typical storage ring photon pulses, the low intensities achievable sets practical limits with this kind of source.

BESSY therefore presented the Technical Design Report for a multi-FEL, multi-user facility in the photon energy range $24 \mathrm{eV}$ to $1000 \mathrm{eV}$, utilizing three independent cascades of HGHG-FEL to frequency convert an fs-seed pulse from an external UV laser to shorter wavelength. This technique assures reproducible radiation pulses controllable down to a few femtoseconds in duration. Further advantage of this approach is the external seed serving as master clock for the synchronization of pump-probe experiments over the hole wavelength range from the UV to soft X-rays.

*Work supported by BMBF and Zukunftsfonds des Landes Berlin "kraemer@bessy.de
Table 1 lists some of the main performance parameters of the BESSY soft X-ray FEL. In combination with a superconducting electron-linac a unique flexible light source will be available able to adapt to any experimental requirement in terms of pulse repetition rate and pulse pattern.

Table 1: Parameters of the BESSY Soft X-ray FEL.

\begin{tabular}{|l|c|c|}
\hline Parameter & Value & Unit \\
\hline No. of FEL lines & 3 & \\
\hline No. of end stations & $9(15)$ & \\
\hline Wavelength range & $51-1.24$ & $\mathrm{~nm}$ \\
\hline Beam peak power & $1.5-14$ & $\mathrm{GW}$ \\
\hline Peak brilliance & $6 \cdot 10^{29}-$ & $\mathrm{ph} / \mathrm{s} \cdot \mathrm{mm}^{2} \cdot \mathrm{mrad}^{2} \cdot 0.1 \% \mathrm{bw}$ \\
\hline Pulse duration & 20 & $\mathrm{fs}$ \\
\hline $\begin{array}{l}\text { Min. pulse } \\
\text { separation }\end{array}$ & 2 & $\mu \mathrm{s}$ \\
\hline Repetition rate & $1-25$ & $\mathrm{kHz}$ \\
\hline Photon beam size & $14-160$ & $\mu \mathrm{m}$ \\
\hline Beam divergence & $37-140$ & $\mu \mathrm{rad}$ \\
\hline Electron energy & 2.3 & $\mathrm{GeV}$ \\
\hline Operation mode & $\mathrm{CW}$ & \\
\hline
\end{tabular}

\section{THE FEL FACILITY}

The FEL facility will be located in close neighbourhood to the BESSY laboratory making effective use of synergies in machine and experiment developments. Fig. 1 gives an architectural view of the future complex.

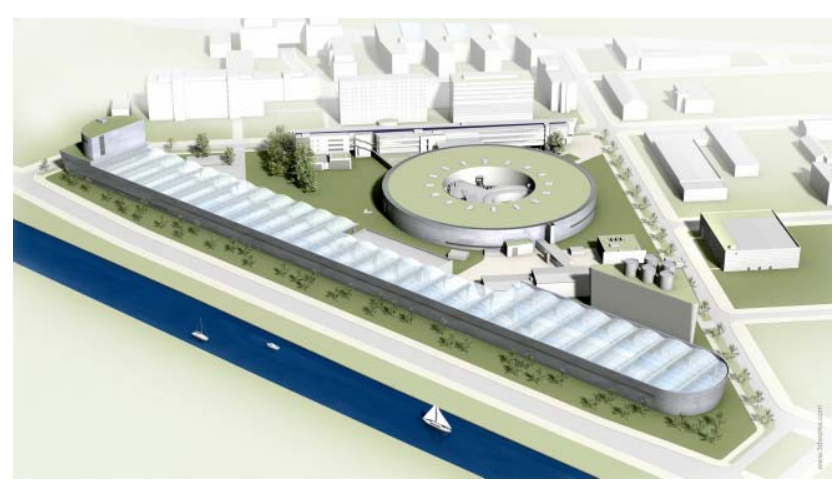

Fig. 1: Aerial view of the proposed BESSY complex with the BESSY II light source circular building, the MLS at right hand and the $450 \mathrm{~m}$ long FEL-building housing linac, the FEL-lines and experimental areas. 


\section{Simulation of Photon Beam Characteristics}

Based on start to end simulations using the codes ASTRA and ELEGANT electron beam phase space distributions were derived and the 3-D FEL-code GENESIS[6] was used in time-dependent mode to calculate the FEL-output pulse and pulse spectral density. Fig. 2 shows a typical result at the last undulator (amplifier) exit of the third cascade of the so-called 'medium energy' FEL spanning photon energies 100 to $600 \mathrm{eV}$. A17 fs (fwhm) Gaussian profile pulse of $500 \mathrm{MW}$ peak power at $\lambda=258 \mathrm{~nm}$ was assumed for seeding the first HGHG-stage.

The output pulse duration reflects the (in principle variable) seed duration while the relative spectral linewidth is about $1 \cdot 10^{-3}$ with two asymmetrically distributed sidebands. The sidebands are originated by overbunched electrons performing synchrotron oscillations in the ponderomotive bucket. The number of sidebands scales with the number of HGHG-stages of the cascade while the asymmetry is caused by the slippage.
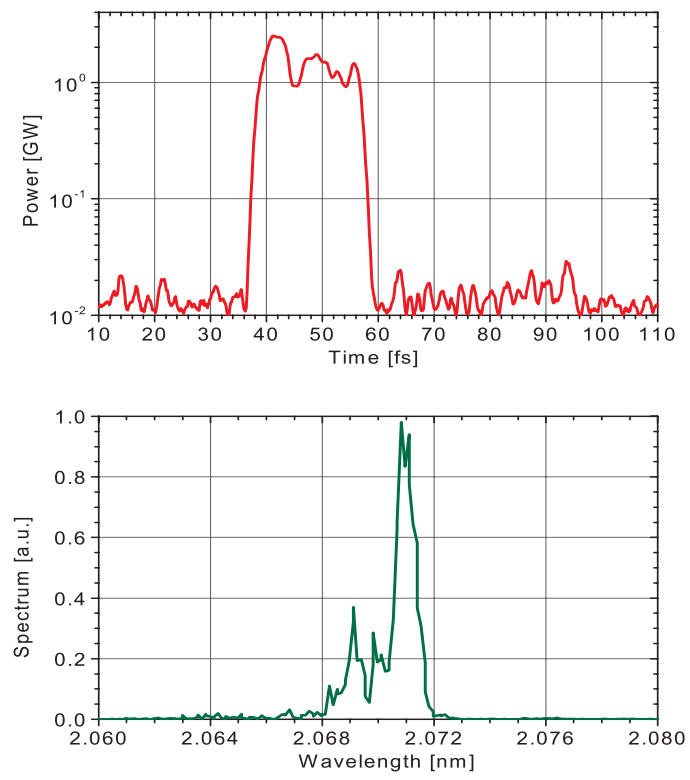

Figure 2: Typical pulse structure (upper graph) and spectral density (lower graph) as calculated for the medium energy FEL line, at $\lambda_{\text {ph }}=2 \mathrm{~nm}$.

Shot noise in the undulators is expected to cause degradation of the coherent properties of the HGHG output especially when operating at very high harmonics as is the case of cascading. The ratio of noise power $P_{n}$ to signal power $\mathrm{P}_{\mathrm{s}}$ at the input (in) and output (out) satisfies the relation

$$
\left(\frac{P_{n}}{P_{s}}\right)_{\text {out }}=n^{2}\left(\frac{P_{n}}{P_{s}}\right)_{\text {in }}
$$

where $\mathrm{n}$ is the harmonic number. For the BESSY-FEL $\mathrm{n}=$ 225 at maximum as the seed laser's wavelength is $280 \mathrm{~nm}$ and the shortest output wavelength $\lambda=1.24 \mathrm{~nm}$. To reproduce the coherence of optical seed pulse at the high power output despite of the high n, a peak-power of the optical seed in the order of $500 \mathrm{MW}$ is required.

Calculation for an output wavelength of $1.24 \mathrm{~nm}$ varying the seed-pulse power from $150 \mathrm{~W}$ to $150 \mathrm{MW}$ in steps of a factor of 10 clearly show the effect of the shotnoise, see fig. 3. Vanishing influence of the noise can be expected only at the highest seed power. A reasonable output signal of $100 \mathrm{MW}$ is achieved for a seed of 1.5 MW peak power, while saturation ( $2 \mathrm{GW}$ output) requires a seed in excess of $150 \mathrm{MW}$. Simulations are based on the BESSY HE-FEL geometry.

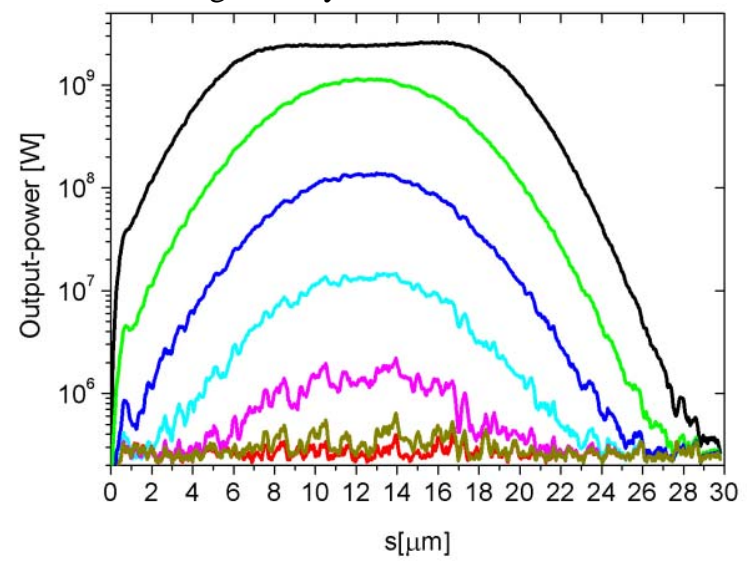

Figure 3: Output pulse power along the electron bunch position at the exit of a $15 \mathrm{~m}$ long undulator for different input seed power. The graphs correspond to a seed power of $\mathrm{P}_{\mathrm{s}}=150 \mathrm{MW}$ (upper black line) successively reduced by a factor of 10 down to $150 \mathrm{~W}$ (lowest red curve).

\section{TESLA Modules for the CW-Linac}

Basis for the linac accelerating sections are TESLA 9cell cavities with eight cavities arranged in a common cryo-module. Minor modification are required to allow for CW-operation of the modules. As a modest accelerating field of $16 \mathrm{MV} / \mathrm{m}$ will be used the cryogenic losses are less than $25 \mathrm{~W} /$ cavity permitting to run the cavities $\mathrm{CW}$ without exceeding the level of capacity of $1.8 \mathrm{~K}$ superfluid $\mathrm{He}$ of $3.6 \mathrm{~kW}$. CW operation mode gives full flexibility to set the machine according to the experimenters needs rather than to provide a fixed pulse pattern at constant repetition rate.

Work at the RF test bench is proceeding. A first $15 \mathrm{~kW}$ klystron amplifier has been developed and is in routine operation for tests at cavities, tuners and couplers; a 10 $\mathrm{kW}$ prototype $1.3 \mathrm{GHz}$ IOT power-amplifier will be available by mid of the year.

\section{The Photoinjector}

The electron beam parameters are of major impact to the FEL characteristics, as the beam emittance has to be smaller than the photon output wavelength $\lambda_{\text {ph }}$ to achieve lasing:

$$
\frac{\varepsilon_{O}}{\gamma} \leq \frac{\lambda_{\mathrm{ph}}}{4 \pi}
$$


where $\varepsilon_{\mathrm{o}}$ is the normalized emittance and $\mathrm{E} / \mathrm{m}_{\mathrm{e}} \mathrm{c}^{2}=\gamma$ with the electron beam energy $E$ and the electron rest mass $m_{e}$.

The PITZ RF-photoinjector[7] now in operation at the DESY VUVFEL already demonstrated the performance as required for the BESSY-FEL injector. However, as the repetition frequency of this injector is $10 \mathrm{~Hz}$, an improved version of the gun cavity was designed to allow for short bunch-trains ( 3 bunches spaced by $2-3 \mu \mathrm{s}$ ) at a repetition frequency of up to $1 \mathrm{kHz}$. This gun will be tested at PITZ.

Recent progress in superconducting RF-injector development stimulated a FZR-BESSY-MBI-DESY collaboration to construct a superconducting 3.5-cell RFinjector[8]. Simulations indicate that with RF-focussing a $1 \mathrm{~mm} \cdot \mathrm{mrad}$ slice emittance can be expected when a long flattop photocathode laser profile is adopted with most realistic rise and fall-times of 4 ps. Similar results were obtained in simulations utilizing the emittance conservation principle.

\section{$R \& D$ at the HoBiCaT-Test Bench}

The HoBiCaT test bench now operational at BESSY is designed to qualify linac components. Feed by a Linde TCF50 LHe refrigerator and equipped with a pumping station providing a cooling capacity of $80 \mathrm{~W}$ at $1.8 \mathrm{~K}$.

Fig. 4 shows a photograph of the bench together with one out of two recently delivered 9-cell cavities manufactured and processed entirely by industry[9]. The cavities exceed the specifications required for the BESSY$\mathrm{CW}$ linac structures in terms of maximum field and unloaded Q-value.

Power tests with the TTF-III coupler confirmed limitations in $\mathrm{CW}$ operation at $10 \mathrm{~kW}$ travelling-wave (TW) and $5 \mathrm{~kW}$ standing-wave (SW) power. Minor modifications improving cooling of the inner conductor indicated that the coupler will operate safely up to $8 \mathrm{~kW}$ SW and suggest that even $25 \mathrm{~kW} \mathrm{SW}$ power should be feasible[10].

As the BESSY low current accelerating structures are dominated by microphonics effective Piezo-dampers, presently under preparation, are needed to reduce RFpower and thus costs for RF-systems and cryoinfrastructure.

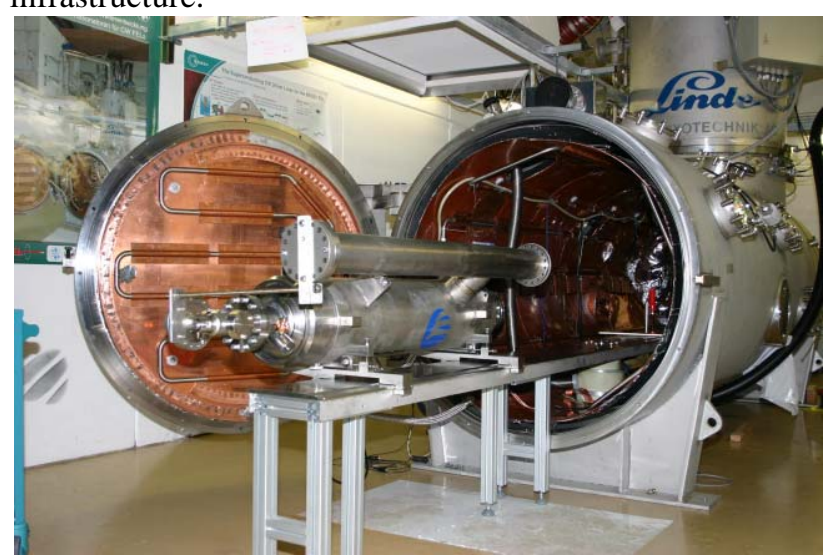

Figure 4: Photograph of the first TESLA type 9-cell cavity produced by industry at the HoBiCaT test bench.

\section{FEL Undulator Sections}

For the FEL-undulator sections various types of insertions (linear and elliptical permanent magnet devices) of different period-length are required. All undulators sum up to a total length of $120 \mathrm{~m}$ for the three FEL-lines. The undulators are segmented with a maximum length of $4 \mathrm{~m}$. For wavelength tuning all undulators are variable in gap-setting. Photon beam polarization is controlled by row-shifts of modified APPLE II permanent magnet IDs to be used as the radiator and the final amplifier in the last HGHG-stage. The design strategies following closely the successful operating IDs at BESSY II[11].

\section{SUMMARY}

The technical design of the BESSY soft X-ray FEL facility shows that the project can be realized with existing technologies. $R \& D$ in the most challenging injector and linac hardware is progressing. Evaluation of the project is scheduled for mid 2005.

\section{ACKNOWLEDGEMENTS}

The help of many colleagues from BESSY and from APS, BNL, Cornell, DESY, FZ-Rossendorf, MBIBerlin and TU Dresden providing valuable input to the BESSY-FEL project is acknowledged.

\section{REFERENCES}

[1] The BESSY Soft X-ray Free Electron Laser, TDR, BESSY March 2004, eds. D. Krämer, E. Jaeschke, W. Eberhardt, ISBN 3-9809534-0-8, Berlin (2004).

[2] I. Ben-Zvi, F. DiMauro, S. Krinsky, L. H. Yu, Nucl. Instrum. And Meth. A304 (1991)181.

[3] R. Klein et al., The Metrology Light Source of the Physikalisch Technische Bundesanstalt in BerlinAdlershof, Proceedings EPAC, Lucerne, 5 to 9 July 2004, p. 273.

[4] Vision of Science: the BESSY SASE-FEL in Berlin Adlershof, BESSY, Berlin 2001.

[5] S. Khan et al., Commissioning Results from the BESSY II Femtoslicing X-Ray Source, these proceedings.

[6] S. Reiche, Nucl. Instrum. and Meth., A429 (1999)243.

[7] M. Krasilnikow et al., Recent Developments at PITZ, this conference

[8] R. Xiang et al., Status of the 3 1/2 Cell Superconductive RF Gun Project in Rossendorf, this conference.

[9] M. Pekeler et al., Performance of TESLA Cavities in Vertical and Horizontal Tests after Fabrication and Preparation in Industry, this conference.

[10]J. Knobloch et al., CW Operation of the TTF III Input Coupler, this conference.

[11] J. Bahrdt et al., Nucl. Instr. Meth. A467(2001)21. 